\title{
Bioimaging for cardiometabolic translational medicine: from cellular messengers to organism function
}

\author{
Rui-Ping Xiao
}

Published online: 28 July 2013

(C) Springer-Verlag Berlin Heidelberg 2013

The twentieth century witnessed two great breakthroughsWatson and Crick's discovery of the double-helix structure of DNA and the central dogma of the flow of genetic information. At the turn of this millennium, the Human Genome Program has led to an explosion of biomedical information and integration of physics, computational science, and engineering, thereby transforming the biomedical landscape and enabling the new biology of the twenty-first century. Understanding the structural and architectural dynamics of living organisms at the cellular and molecular levels has become a prime focus of biomedical research in the postgenomic era. The discipline of biomolecular imaging holds great promise for visualizing activities of biological systems in ever finer details while simultaneously obtaining multidimensional, multifaceted, panoramic views of the system as a whole. Biomolecular imaging is one of the main forces to facilitate a seamless unification of the reductionist approach with integrative translational medicine of the twenty-first century biology era.

Over the past two decades, enormous progress has been made in the development of molecular and cellular bioimaging instrumentation and methodology, not only revealing biological building blocks and signaling paradigms, but also identifying novel functions and malfunctions of genes and proteins. In particular, advances in modern fluorescence microscopy have been noteworthy, including the development of a plethora of small molecule probes or naturally fluorescent proteins (notably the green fluorescent protein) as well as engineered fluorescent proteins. In synchrony with the advances of modern photonics, optical engineering, as well as computational and material sciences, generations of fluorescence microscopes

R.-P. Xiao $(\bowtie)$

Institute of Molecular Medicine, State Key Laboratory of Biomembrane and Membrane Biotechnology, Beijing Key

Laboratory of Cardiometabolic Molecular Medicine,

Peking-Tsinghua Center for Life Sciences,

Peking University, Beijing 100871, China

e-mail: Xiaor@pku.edu.cn have provided for ultrafast, super-resolution views at the molecular, cellular, tissue, organ, and even living animal level. Bioimaging will undoubtedly open up new avenues in elucidating the nature of diseases and lead the way to therapeutic triumphs - successful detection, prevention, intervention, and treatment of complex diseases such as heart failure and diabetes.

In this special issue on "Biomolecular imaging on cardiometabolic systems" three excellent review articles, contributed by experts in the field of cardiometabolic physiology and biophysics, provide timely and comprehensive updates and perspectives on bioimaging of crucial cellular signaling events, (1) calcium $\left(\mathrm{Ca}^{2+}\right),(2)$ reactive oxygen species (ROS), and (3) beta-cell insulin secretion. Among all known second messengers, $\mathrm{Ca}^{2+}$ is the most evolutionarily conserved, universal, and versatile. The search for the principles governing the spatiotemporal signaling selectivity of $\mathrm{Ca}^{2+}$ has fascinated biologists, chemists, and physicists for more than a century, leading to the discovery of the elementary $\mathrm{Ca}^{2+}$ release event, the so-called $\mathrm{Ca}^{2+}$ spark [1], and the identification of $\mathrm{Ca}^{2+}$-binding proteins, such as calmodulin $(\mathrm{CaM})$ and $\mathrm{Ca}-\mathrm{CaM}$-dependent protein kinase II (CaMKII) [2], and their crucial roles in compartmentalizing $\mathrm{Ca}^{2+}$-dependent signaling. The review by Bossuyt and Bers not only provides a cutting-edge picture of the recent technological advances enabling the study of subcellular $\mathrm{Ca}^{2+}$-CaM-CaMKII signaling events in the normal and the failing heart, but also charts the future directions and outstanding challenges in the field [3].

Equally appealing, ROS act as essential cellular messengers. Too important to live without them, ROS are involved in vital biological processes such as metabolism, immune response and aging. But when in excess, ROS evoke pathological oxidative stress that has been implicated in the pathogenesis of metabolic and cardiovascular diseases, including heart failure and diabetes. In contrast to the deceptive simplicity of $\mathrm{Ca}^{2+}$ signaling in terms of design principles [1], ROS signaling is notoriously complex. All ROS are not created equal; they 
cover the full spectra of "colors" and "flavors." The current challenge in the field of ROS signaling is how to spatiotemporally visualize and quantify local and global ROS dynamics as a humble beginning to unravel the seemingly formidable ROS signaling pathways. Using multidisciplinary approaches, highly selective and sensitive synthetic probes, and genetically encoded fluorescent protein-based ROS indicators enables the field to make important breakthroughs in ROS imaging in the setting of normal physiology and abnormal disease conditions. For benchscientists and physician-scientists to glean a historical perspective and an updated landscape of the field, the review by Wang et al. is an excellent source of both information and inspiration. It provides a current view of ROS imaging and envisions areas of challenges and translational potential [4].

The final aspect of this special issue is focused on imaging beta-cell mass and function in situ and in vivo [5]. The review by Yang et al. summarizes recent progresses of beta-cell imaging techniques and underscores pressing challenges in the field [5]. It has been well accepted that loss of pancreatic beta-cell mass or function plays a pivotal role in the development of diabetes, a global epidemic afflicting 347 million people world-wide [6]. Furthermore, recent studies have demonstrated that more than $80 \%$ of diabetes deaths are attributed to cardiovascular complications, including heart disease, stroke, retinopathy, and kidney disease. Thus, metabolic and cardiovascular diseases are interwoven chronic conditions that have become the most serious threats to human health [6]. High-resolution fluorescence imaging techniques provide novel insights into the fundamental mechanisms underlying glucose-induced insulin secretion in individual beta-cells and isolated pancreatic islets. In addition, other molecular imaging tools can even provide information on beta-cell mass and function in living animals or patients with diabetes.

What is truly remarkable about biomolecular imaging is the rapid translation of bench work to the bedside in addition to the rapid development of paradigms as knowledge has been assimilated. All three reviews have highlighted potential clinical applications of imaging studies of $\mathrm{Ca}^{2+}$-CaM-CaMKII, ROS, and beta-cell mass and function in disease diagnosis and treatment. Since ingenious multidisciplinary techniques are continuously invented and innovatively integrated, we enthusiastically look forward to a more vigorous and rigorous field of biomolecular imaging in the era of translational medicine.

\section{References}

1. Cheng H, Lederer WJ (2008) Calcium sparks. Physiol Rev 88:14911545

2. Bers DM (2008) Calcium cycling and signaling in cardiac myocytes. Annu Rev Physiol 70:23-49

3. Bossuyt J, Bers DM (2013) Visualizing CaMKII and CaM activity: a paradigm of compartmentalized signaling. J Mol Med. doi:10.1007/ s00109-013-1060-y

4. Wang X, Fang H, Huang Z, Shang W, Hou T, Cheng A, Cheng H (2013) Imaging ROS Signaling in Cells and Animals. J Mol Med. doi:10.1007/s00109-013-1067-4

5. Yang L, Ji W, Xue Y, Chen L (2013) Imaging beta-cell mass and function in situ and in vivo. J Mol Med. doi:10.1007/s00109-013-1056-7

6. Danaei G, Finucane MM, Lu Y, Singh GM, Cowan MJ, Paciorek CJ et al (2011) National, regional, and global trends in fasting plasma glucose and diabetes prevalence since 1980: systematic analysis of health examination surveys and epidemiological studies with 370 country-years and 2.7 million participants. Lancet 378:31-40 